\title{
¿Cuál es el Efecto de la Moringa oleifera sobre la Dinámica Ruminal? Revisión sistemática
}

\author{
What is the Effect of Moringa oleifera on the Ruminal Dynamics? \\ A Systematic Review \\ Indira Isis García Q. ${ }^{13}$, Jairo Mora-Delgado ${ }^{1}$, Julián Estrada $\mathrm{A}^{2}$, Roberto Piñeros $\mathrm{V}^{1}$
}

\section{Resumen}

Moringa oleifera es un árbol con alto potencial para suplir deficiencias alimenticias en sistemas productivos tropicales, especialmente en rumiantes, ya que cuenta con aceptables valores nutricionales, buena palatabilidad, adaptabilidad a condiciones agrestes y alta producción de biomasa. El presente estudio es una revisión sistemática sobre el efecto del consumo de $M$. oleifera en la dinámica ruminal. Se planteó un protocolo de búsqueda que permitió sistematizar el proceso para el rastreo de información. La búsqueda identificó trabajos sobre el efecto del consumo de forraje de esta planta sobre la dinámica ruminal, en cuanto a digestibilidad y degradabilidad de la materia seca, producción de gases y cambios en población microbiana. Se consultaron 17 bases de datos bibliográficas, obteniéndose 28 artículos y 15 calificados como de calidad aceptable para el tema del estudio. Se concluye que hay una alta variación en los resultados publicados, lo cual está relacionado con la diversidad de las condiciones en que se desarrollan los estudios, el material empleado y las mezclas con forrajes y concentrados, así como las metodologías y técnicas utilizadas.

Palabras clave: alimentación animal, revisión sistemática, rumiante, poblaciones microbianas

\section{Abstract}

Moringa oleifera is a tree with high potential to meet nutritional deficiencies in tropical production systems, especially in ruminants due to its nutritional value, palatability, biomass production and adaptability to harsh conditions. This systematic

\footnotetext{
${ }^{1}$ Grupo de investigación Sistemas Agroforestales Pecuarios, Facultad de Medicina Veterinaria y Zootecnia, Universidad del Tolima, Ibagué (Tolima), Colombia

${ }^{2}$ Grupo de Investigación Biología de la Producción Pecuaria, Universidad de Caldas, Manizales (Caldas), Colombia

${ }^{3}$ E-mail: igarcia@ut.edu.co
}

Recibido: 23 de abril de 2016

Aceptado para publicación: 8 de octubre de 2016 
review aimed to highlight the effect of fodder consumption in the ruminal dynamics. A search protocol was implemented to systematize the process for tracking the information. The search identified studies related to the effect of consumption of $M$. oleifera on rumen dynamics in terms of digestibility and degradability of dry matter, production of gases and changes in microbial population. Seventeen bibliographic databases were searched yielding 28 articles and 15 rated as acceptable quality for the purpose of this study. It was concluded that there was a high variability in the results published which is related to the diversity of conditions in the studies, material used and mixtures with other forages and concentrates, as well as the methodologies and techniques used.

Key words: animal feeding, systematic review, ruminant, microbial population

\section{INTRODUCCIÓN}

La literatura reporta diversos centros de origen de Moringa oleifera Lam (Brassicales: Moringaceae), pero todos coinciden en el Oriente Medio; así el sur del Himalaya, noreste de India, Pakistán, Bangladesh, Arabia Saudita y Afganistán, habiéndose naturalizado en la mayoría de países tropicales (Foidl et al., 1999; Carballo, 2011). El árbol se caracteriza por tener una altura entre 10-12 m, tronco leñoso y recto con diámetro de $20-40 \mathrm{~cm}$, siendo una especie de rápido crecimiento y puede vivir hasta 20 años (Falasca, 2008). Asimismo, posee copa abierta tipo paraguas y alta resistencia a plagas y enfermedades (Pérez et al., 2010). La importancia del uso de esta planta como especie forrajera se debe a sus características nutricionales y alta producción de biomasa fresca (Reyes, 2006; Pérez et al., 2010).

M. oleifera ha sido ampliamente estudiada en el área de alimentación y medicina humana. Las cualidades nutritivas de las hojas están entre las mejores de los vegetales perennes, pues presenta $27 \%$ de proteína cruda y cantidades importantes de calcio, hierro, fósforo, y vitamina A y C. Las hojas de moringa pueden cosecharse durante la época seca, cuando no hay otros vegetales frescos disponibles (Folkard y Sutherland, 1996).
El cultivo de $M$. oleifera se está extendiendo en América, desde el sur de los EEUU hasta el norte de Chile y Argentina (Falasca y Bernabé, 2008). Se cree que fue introducida en América por el intercambio de plantas realizado por los españoles mediante los bar$\cos$ que realizaban comercio entre Filipinas y México, habiéndose encontrado referencias a esta especie en envíos de 1782, 1793, 1797 y 1872 (Pacheco, 2006). En Centroamérica fue introducida como especie ornamental y, para cercos vivos, en la década de 1920 (Foidl et al., 1999). Por otro lado, no se dispone de información sobre su introducción en Colombia.

En las últimas dos décadas se han realizado investigaciones tendientes a la identificación de principios activos y sus mecanismos de acción, lo que ha permitido explicar muchos de los efectos benéficos previamente conocidos, optimizar su explotación y proponer nuevas aplicaciones. No obstante, aún se requiere la confirmación científica de algunos usos (Martín et al., 2013).

Dada la importancia nutricional como económica de esta especie, el presente estudio tuvo como objetivo conocer, mediante una revisión sistemática, el grado de avance de los estudios del efecto de la M. oleifera sobre la dinámica ruminal. 


\section{Materiales y Métodos}

Los estudios sobre $M$. oleifera fueron seleccionados de acuerdo al protocolo y las recomendaciones de PRISMA (http:// www.prisma-statement.org/) para el desarrollo de una revisión sistemática (RS) y el manual de Cochrane (Higgins y Green 2011); así como los reportes de Gómez Ortega et al. (2013) y Ferreira et al. (2011). En la estrategia de búsqueda se incluyeron 17 bases de datos bibliográficas específicas del área agropecuaria: University of Chicago Journals, AGBios, Embrapa, Academic Search Complete, Agris, Scielo, Science Direct, Agricola, Pubmed, Journal of Animal Science, Animal Production, Journal of Dairy Science, Springer Journal, Scopus, Springer Books, e-books y EBSCO. Además, se consultaron diversos documentos, tesis de grado del repositorio de la Universidad Nacional de Colombia y documentos publicados en buscadores académicos. No se restringieron estudios por año ni por idioma y se tomaron los documentos publicados a diciembre de 2014.

La búsqueda se diseñó siguiendo la siguiente ruta: 1) Se definió a los rumiantes (bovinos, caprinos, ovinos) como la población objetivo, 2) el proceso a evaluar fue el efecto en la dinámica ruminal, 3) los resultados medidos en los estudios fueron el efecto de $M$. oleifera sobre la dinámica ruminal, digestibilidad y degradabilidad de la materia seca, la producción de gases y los cambios en la población microbiana.

En la búsqueda se incluyeron estudios de publicaciones de carácter científico, revisiones narrativas, conferencias y tesis de grado, donde se haya utilizado M. oleifera en la alimentación de rumiantes. Los términos de búsqueda, en español e inglés, utilizados fueron una combinación de Moringa oleifera con ruminants*, sheep, goats, cattle, bovine, animal nutrition, o marango. Se utilizaron los conectores 'or', 'and' y '*'; donde este últi- mo realiza búsquedas orientadas por la raíz de la palabra o palabras relacionadas.

Se excluyeron los estudios en los que hacían referencia a especies diferentes a rumiantes, estudios no realizados en nutrición y estudios de carácter no científico. En la búsqueda inicial se incluyeron todos los documentos arrojados por las bases de datos, según los criterios de exclusión y selección contenidos en título y resumen. Posteriormente se excluyeron artículos repetidos, considerados duplicados por encontrarse en diferentes bases de datos.

Los criterios de inclusión fueron artículos relevantes (aquellos que son mayormente citados por otros autores). Se constató que las referencias bibliográficas citadas por otros autores se hayan incluido en la búsqueda. Los documentos fueron revisados teniendo en cuenta que en su metodología mencionaran la especie animal (rumiantes), digestibilidad in vitro, in vivo o in situ, microorganismo ruminal o producción de gases. Luego se excluyeron los manuales técnicos, evaluaciones nutricionales (composicional), trabajos de aceptabilidad y pruebas de consumo.

Al final, se desarrolló un formulario de evaluación para la RS modificando la metodología descrita por Ferreira (2011). Se tuvieron en cuenta los 14 ítems, que se encuentran clasificados en tres secciones donde se incluye el denominado IMRD (Introducción, metodología, resultados, discusión). Adicional a este, se tuvo en cuenta el título y el resumen (Cuadro 1). El proceso de selección, exclusión e inclusión se ilustra en la Figura 1.

Se contó con la colaboración de tres expertos en el área. Para el presente estudio se seleccionaron los artículos que estuvieran por encima de 10 puntos, que corresponde a aquellos que presentaban clara y apropiadamente los resultados. 
Cuadro 1. Criterios de selección por formulario de evaluación en la revisión sistemática sobre estudios de la dinamica ruminal con Moringa oleifera ${ }^{1}$

\begin{tabular}{llc}
\hline Criterio & Ítem & Puntaje \\
\hline Introducción & Pertinencia del título & 1 \\
& Calidad del resumen & 1 \\
& Objetivos del estudio & 1 \\
& Justificación & 1 \\
& Evaluación de la introducción y marco teórico & 1 \\
Metodología & Instrumentos de medición & 1 \\
& Diseño & 1 \\
& Muestra & 1 \\
& Procedimiento de recolección y análisis de datos & 1 \\
Resultados & El estudio tiene asignación aleatoria & 1 \\
& Presentación de resultados & 1 \\
& Discusión, conclusión y recomendaciones & 1 \\
& Referencias & 1 \\
\hline Total & Número de veces que ha sido citado & 1 \\
\hline
\end{tabular}

${ }^{1}$ Si el resultado es positivo se suma 1 punto y si es negativo se resta 1 punto.

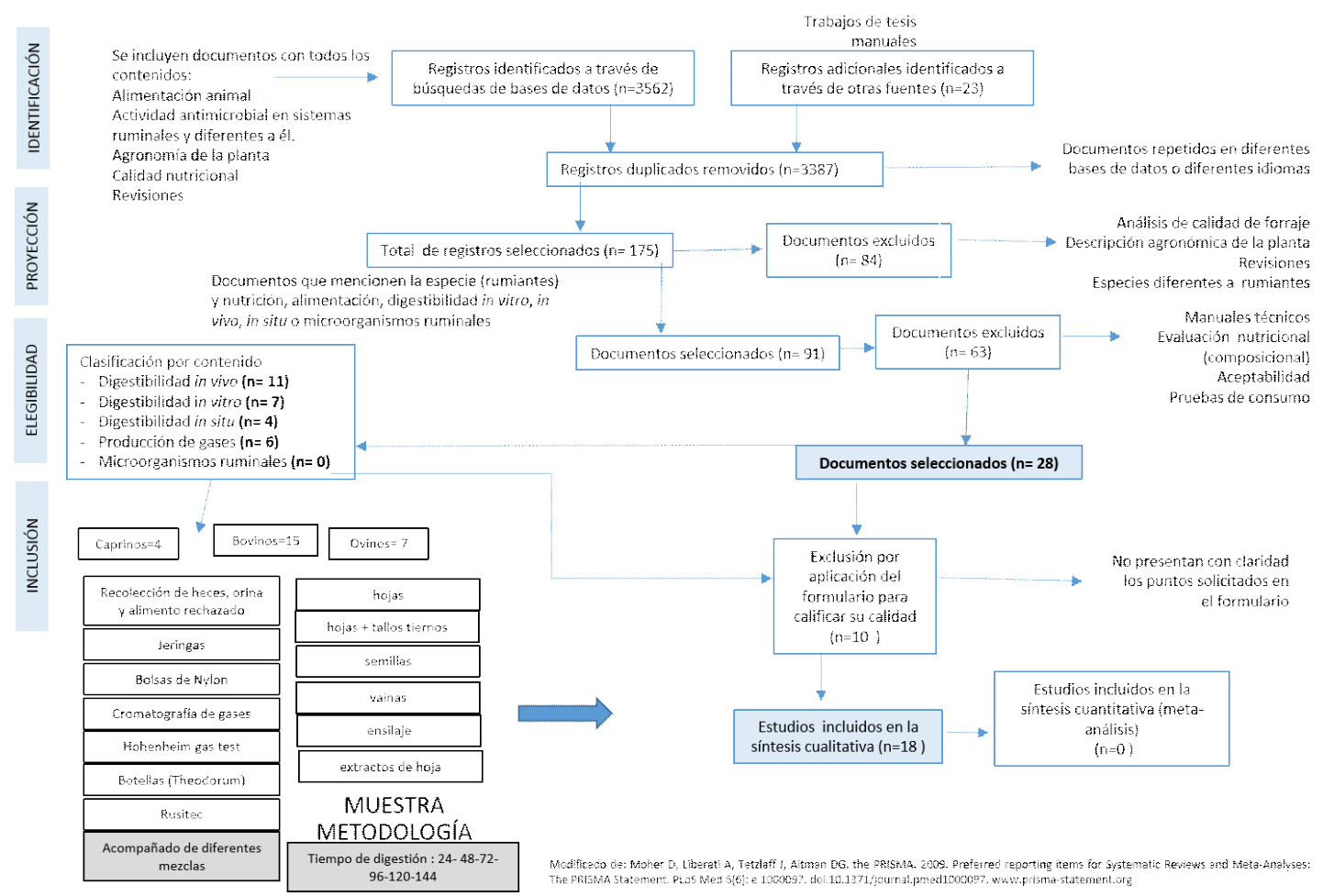

Figura 1. Diagrama de flujo de la revisión sistematica sobre estudios nutricionales para rumiantes con Moringa oleifera 


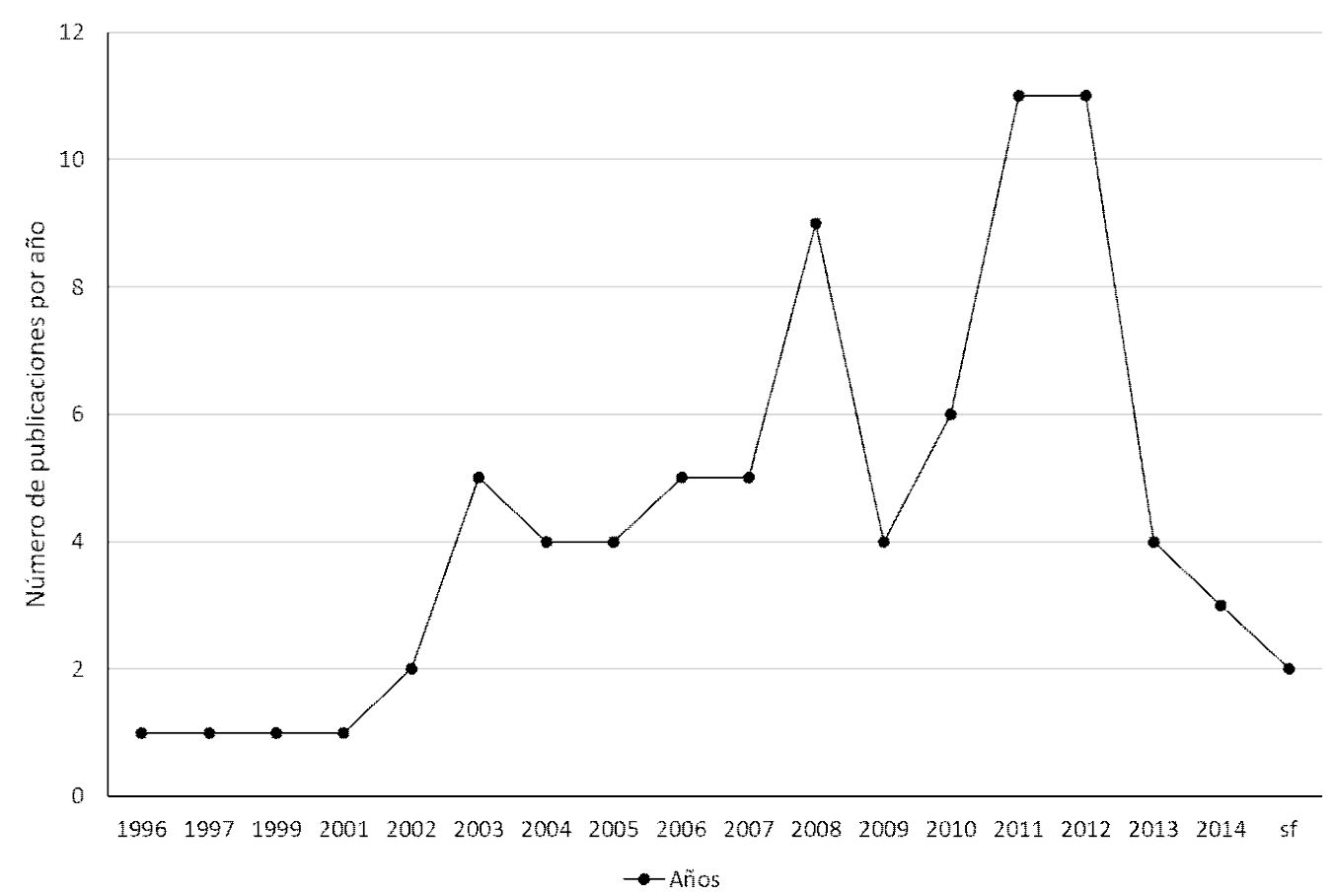

Figura 2. Distribución en el tiempo de estudios relacionados con el consumo de Moringa oleifera en rumiantes desde 1996 hasta 2014

\section{Resultados y Discusión}

Se trabajó con 28 publicaciones seleccionadas, cuya distribución según el año de publicación se muestra en la Figura 2. El mayor número de estudios sobre el tema se realizó en 2011 y 2012, con 11 publicaciones cada año. Asimismo, se observa un aumento de la producción intelectual en los últimos 10 años. Entre los estudios previos a 1996, destacan los dirigidos a la descripción botánica de la planta, sobresaliendo por su sistematicidad el artículo de Ramachandran et al. (1980).

En el Cuadro 2 se presentan las 28 referencias asociadas a los efectos de $M$. oleifera en la dinámica ruminal. Así mismo, se indica la especie de rumiante involucrada y la puntuación obtenida en la evaluación de selección. La especie animal más usada en los estudios fue la bovina con 16 estudios, seguida de la caprina y ovina con 7 y 5 estudios, respectivamente. Trece de estas tuvieron una calificación igual o menor de 10 , de allí que se les descartó del análisis siguiente.

Del listado de 28 estudios, al aplicar el formulario para medir la calidad, se seleccionaron 14 estudios que reunían, a criterio de los evaluadores, las características requeridas de un artículo científico y, además, contenían la información de interés para este estudio.

Los factores asociados al efecto del consumo de la Moringa oleifera sobre la dinámica ruminal se pueden determinar por indicadores como digestibilidad de la materia seca (DMS), contenido de fibra detergente neutra (FDN), proteína cruda (PC) y parámetros de fermentación como la producción de gases ruminales. 
Cuadro 2. Referencias asociadas al efecto de la Moringa oleifera sobre la dinámica ruminal

\begin{tabular}{|c|c|c|c|c|}
\hline Referencia & Especie & Autor & Año & Puntaje \\
\hline 1 & Caprina & Moyo et al. & 2014 & 9 \\
\hline 2 & Bovina & Rodríguez & 2011 & 9 \\
\hline 3 & Caprina & Lam Kim Yen et al. & 2007 & 9 \\
\hline 4 & Bovina & Hoffmann et al. & 2003 & 9 \\
\hline 5 & Ovina & Adegun et al. & 2011 & 9 \\
\hline 6 & Ovina & Astuti et al. & 2011 & 10 \\
\hline 7 & Ovina & Jelali y Ben Salem & 2014 & 10 \\
\hline 8 & Caprina & Luu Huu Manh et al. & 2005 & 10 \\
\hline 9 & Caprina & Montejo et al. & 2012 & 10 \\
\hline 10 & Bovina & Makkar y Becker & 1996 & 10 \\
\hline 11 & Ovina & Murro et al. & 2003 & 10 \\
\hline 12 & Ovina & Patra & 2010 & 10 \\
\hline 13 & Bovina & Sánchez et al. & 2006 & 10 \\
\hline 14 & Bovina & Nouala et al. & 2006 & 10 \\
\hline 15 & Caprina & Aregheore & 2002 & 11 \\
\hline 16 & Bovina & Gutiérrez & 2012 & 11 \\
\hline 17 & Bovina & Men dieta-Araica & 2011 & 11 \\
\hline 18 & Bovina & Reyes & 2006 & 11 \\
\hline 19 & Caprina & Sarwatt et al. & 2002 & 11 \\
\hline 20 & Bovina & Sarwatt et al. & 2004 & 11 \\
\hline 21 & Bovina & Al-Masri & 2003 & 12 \\
\hline 22 & Bovina & Melesse et al. & 2013 & 12 \\
\hline 23 & Bovina & Pedraza et al. & 2013 & 12 \\
\hline 24 & Bovina & Almanza et al. & 2013 & 12 \\
\hline 25 & Bovina & Soliva et al. & 2005 & 12 \\
\hline 26 & Bovina & Alexander et al. & 2008 & 13 \\
\hline 27 & Bovina & Rodríguez et al. & 2014 & 13 \\
\hline 28 & Ovina & Ben Salem y Makkar & 2009 & 14 \\
\hline
\end{tabular}

Como se puede observar en el Cuadro 3 , los valores de degradabilidad reportada por la literatura son variados, en parte debido al diseño propio de los estudios. Por ejemplo, los estudios de Aregeore (2002) y Sarwatt et al. (2002) en caprinos utilizan la misma metodología (in vivo, y la técnica de colecta total de heces), pero con diferente nivel de in- clusión de $M$. oleifera, de allí que la digestibilidad fue de 58.3 a $52.1 \%$ en el primer estudio y de 57.8 a $59.6 \%$ en el segundo. En bovinos, donde se encontró la mayor cantidad de estudios, se emplearon diversas técnicas y fracciones de plantas, así como tiempos de digestión. Por ejemplo, en el estudio de Gutiérrez (2012), utilizando forraje de 
Cuadro 3. Factores asociados al efecto de la Moringa oleifera sobre la dinámica ruminal ${ }^{1}$

\begin{tabular}{|c|c|c|c|c|c|c|c|}
\hline Ref & Especie & Fracción & Método & Técnica & $\begin{array}{l}\text { Digest. / Prod } \\
\text { de gases }\end{array}$ & $\begin{array}{l}\text { Carbohidratos } \\
\text { estructurales }\end{array}$ & $\begin{array}{l}\text { Proteína } \\
\text { cruda }\end{array}$ \\
\hline 15 & Caprina & $\begin{array}{l}\text { Hojas }+ \\
\text { pasto }\end{array}$ & in vivo & $\begin{array}{l}\text { Colecta total } \\
\text { de heces }\end{array}$ & $\begin{array}{l}\text { DMS } 58.3 \text { a } \\
52.1 \% \text { con } \\
\text { moringa }(20- \\
80 \%)\end{array}$ & $\begin{array}{l}\text { FDN } 35.8 \% \text { a } \\
22.6 \% \text { con } \\
\text { Moringa } 20-80 \%\end{array}$ & $\begin{array}{l}\text { PC } 10.5 \text { a } \\
17.1 \% \text { con } \\
\text { moringa de } \\
20 \text { a } 80 \%\end{array}$ \\
\hline 16 & Bovina & Forraje & in situ & $\begin{array}{l}\text { Bolsa de } \\
\text { nylon }\end{array}$ & $\begin{array}{l}\text { DMS } 37.4 \text { a } \\
64.9 \% \text { a las } 48 \\
\text { y } 120 \mathrm{~h}\end{array}$ & FDN $28.8 \%$ & PC $26.7 \%$ \\
\hline 17 & Bovina & Hoja & in vivo & $\begin{array}{l}\text { Colecta total } \\
\text { de heces }\end{array}$ & DMS 74\% & FDN $16.1 \%$ & PC $29.2 \%$ \\
\hline 18 & Bovina & Hojas & in vitro & $\begin{array}{l}\text { Digest. de } \\
\text { dos fases }\end{array}$ & DMS 70.9\% & FDN $28.9 \%$ & PC $22.8 \%$ \\
\hline 19 & Caprina & $\begin{array}{l}\text { Hojas }+ \\
\text { suplemento }\end{array}$ & in vivo & $\begin{array}{l}\text { Colecta total } \\
\text { de heces }\end{array}$ & $\begin{array}{l}\text { DMS } 57.8 \text { a } \\
59.6 \% \text { con } \\
\text { inclusiones del } \\
9 \text { y } 36 \%\end{array}$ & $\begin{array}{l}\text { FDN } 53 \text { y } 36.7 \% \\
\text { con inclusiones de } \\
9 \text { y } 36 \%\end{array}$ & $\begin{array}{l}\text { PC } 16.1 \% \\
\text { con } \\
\text { inclusiones } \\
\text { de } 9 \text { y } 36 \%\end{array}$ \\
\hline 20 & Bovina & Hojas & in situ & $\begin{array}{l}\text { Bolsas de } \\
\text { nylon }\end{array}$ & $\begin{array}{l}\text { DMS } 82 \% \text { a las } \\
48 \mathrm{~h}\end{array}$ & FDN 32\% & PC $27.4 \%$ \\
\hline 21 & Bovina & Forraje & in vitro & $\begin{array}{l}\text { Digest. de } \\
\text { dos fases }\end{array}$ & DMS $84.9 \%$ & FDN $19.3 \%$ & PC $25.5 \%$ \\
\hline 22 & Bovina & $\begin{array}{l}\text { Hojas y } \\
\text { vainas } \\
\text { verdes }\end{array}$ & Gases & $\begin{array}{l}\text { Hohenheim } \\
\text { in vitro gas }\end{array}$ & $\begin{array}{l}40.6-25.1 \\
(\mathrm{ml} / 200 \mathrm{mg} \\
\mathrm{DM})-\mathrm{DMO} \mathrm{de} \\
72.5 \% \text { a } 51.0 \mathrm{a} \\
24 \mathrm{~h}\end{array}$ & FDN $53.1 \%$ & PC $15.8 \%$ \\
\hline 23 & Bovina & Forraje & Gases & Jeringas & $\begin{array}{l}72.7 \mathrm{ml} / 500 \mathrm{mg} \\
\text { de } \mathrm{MS}\end{array}$ & & \\
\hline 24 & Bovina & Forraje & in situ & $\begin{array}{l}\text { Bolsas de } \\
\text { nylon }\end{array}$ & DMS $73.2 \%$ & FDN $30.7 \%$ & PC $22.6 \%$ \\
\hline 25 & Bovina & Hoja & in vitro & Rusitec & $\begin{array}{l}277.9 \mathrm{ml} / 500 \\
\mathrm{mg} \text { de } \mathrm{MS}\end{array}$ & FDN $16.7 \%$ & PC $32.1 \%$ \\
\hline 26 & Bovina & Hojas & Gases & Jeringas & $\begin{array}{l}88.3 \mathrm{ml} / 500 \mathrm{mg} \\
\text { de } \mathrm{MS}\end{array}$ & FDN 39.9\% & PC $23.7 \%$ \\
\hline 21 & Bovina & Forraje & Gases & Jeringas & $\begin{array}{l}83.6 \mathrm{ml} / 500 \mathrm{mg} \\
\text { de } \mathrm{MS}\end{array}$ & FDN 19.3\% & PC $25.5 \%$ \\
\hline 28 & Bovina & Forraje & in vitro & $\begin{array}{l}\text { Botellas de } \\
\text { vidrio } \\
\text { (Theodorou) }\end{array}$ & $\begin{array}{l}67.7 \mathrm{ml} / 500 \mathrm{mg} \\
\text { de } \mathrm{MS}\end{array}$ & FDN $40.5 \%$ & PC $22.2 \%$ \\
\hline 29 & Ovina & $\begin{array}{l}\text { Harina de } \\
\text { semilla }\end{array}$ & in vivo & $\begin{array}{l}\text { Colecta total } \\
\text { de heces }\end{array}$ & DMS 67.4\% & FDN $10.5 \%$ & PC $59.2 \%$ \\
\hline
\end{tabular}

${ }^{1}$ Forraje: mezcla de hojas, pecíolos y tallos hasta $5 \mathrm{~mm}$ de diámetro. Fracción: fracción de la planta utilizada. Método: metodología emplea da en el estudio 
moringa (hojas + ramas + tallos tiernos), la metodología in situ con la técnica de bolsa de nylon y tiempos de 3 a 120 horas de digestión encontró rangos de digestibilidad DMS de 37.4 a $64.9 \%$, mientras que Sarwatt et al. (2004), con la misma metodología y técnica, en mezclas con Pennisetum purpureum, salvado de maíz y torta de algodón, e inclusiones de solo hojas de moringa al 30\%, a 48 horas de digestión, reporta una DMS de $82 \%$.

Se encontró que las mezclas de forrajes constituyen una fuente de variación en la calidad bromatológica. Así se tienen como ejemplo el estudio de la mezcla de $M$. oleifera con pasto henificado de Aregheore (2002) en cabras, el estudio de la mezcla con suplementos (torta de semillas de girasol y salvado de maíz) de Sarwatt et al. (2002), también en caprinos o con el uso de P. purpureum y concentrado comercial en bovinos de Sarwatt et al. (2004) (Cuadro 3).

Los valores reportados en el Cuadro 3, tanto para las fracciones de $M$. oleifera como sus mezclas, revelan el papel que juega la FDN en la digestibilidad de la materia seca de la dieta, encontrando que la digestibilidad disminuye a medida que aumenta los valores de FDN. Ejemplos de esto son los valores reportados de DMS de 67.4\% y FDN de $10.5 \%$ (Ben Salem y Makkar, 2009); de DMS de $82 \%$ y FDN de $32 \%$ (Sarwatt et al., 2002), y de DMS de $73.2 \%$ y FDN de $30.7 \%$ (Almanza et al., 2013).

Se observa que entre las metodologías más usadas para determinar la digestibilidad se encuentran la colecta total de heces, reportada en cuatro estudios, seguido de la técnica de bolsa de nylon con tres estudios y, en menor medida, la técnica de digestibilidad en dos fases con dos estudios. Por otro lado, la técnica de jeringas en la evaluación de gases fue la más empleada (cuatro reportes).

Para la producción de gases, se encuentran los valores reportados por Melesse et al. (2013), comparando fracciones de la plan- ta (hojas y vainas verdes), donde la producción de gas a 24 h varía de $40.6(\mathrm{ml} / 200 \mathrm{mg}$ DM), con DMO de $72.5 \%$ en hojas a 25.1 (ml/200 mg DM) en vainas con una DMO de $51.0 \%$, utilizando liquido ruminal bovino.

Melesse et al. (2013) afirman que, usualmente, la baja producción de gas indica baja degradabilidad en el rumen, pero alimentos con alto contenido de PC producen menos gas durante la fermentación, incluso si su grado de degradación es alto. Esto se debe a que la fermentación de proteínas produce amoníaco, que influye en el carbonato tampón de equilibrio mediante la neutralización de los iones $\mathrm{H}^{+}$desde ácidos grasos volátiles sin liberación de dióxido de carbono (Cone y Van Gelder, 1999).

Según Makkar y Becker (1996), aproximadamente el $24 \%$ de la PC de hojas de $M$. oleifera era soluble en tampón de fosfato $(\mathrm{pH}$ 7, $0.05 \mathrm{M})$. Aparte de esto, la baja producción de gas de las hojas de $M$. oleifera se podría atribuir a la reducción del contenido de carbohidratos no fibrosos (NFC). Getachew et al. (2004) reportaron que la NFC fue positiva, sugiriendo que la producción de gas a partir de la fermentación de hidratos de carbono es relativamente alta en comparación con la fermentación de proteínas. Por otra parte, el bajo volumen de gas de las hojas de M. oleifera podría explicarse por su alto contenido de grasa, lo cual contribuye a bajar la producción de gas (Akinfemi et al., 2009).

Igualmente, Melesse et al. (2013) reportan que las vainas verdes tienen un volumen de gas muy bajo, comparado con las hojas, probablemente debido a su alto contenido de lignina. Se ha informado que el alto contenido de lignina y otras sustancias fibrosas en un alimento puede disminuir la tasa de producción de gas (Getachew et al., 2000; Melesse et al., 2009). El efecto negativo de lignina se puede atribuir a la obstrucción física de los hidratos de carbono estructurales como celulosa y hemicelulosa y a un ataque 
limitado por microorganismos sobre este sustrato. El bajo volumen de producción de gas para las vainas verdes podría ser también causado por la presencia de factores antinutricionales, probablemente taninos y saponinas (Frutos et al., 2002).

Existen diversos factores que generan variabilidad en los resultados de las pruebas nutricionales (Pérez et al., 2013). Cornelissen et al. (2003) reportan variaciones en la calidad nutricional de diferentes plantas leñosas, entre ellas la $M$. oleifera, teniendo en cuenta los rasgos funcionales de las plantas (PFT), enfatizando en respuestas a factores ambientales como agua, nutrientes y sombra, entre otros. Asimismo, Reich et al. (2003) definen estos PFT como cualquier atributo de respuesta con influencia significativa en el establecimiento, supervivencia y capacidad de expresión de las plantas. Estas variaciones determinan rasgos físicos y nutricionales que afectan la preferencia bovina. La variación considerable en las propiedades nutrimentales de la moringa es considerable y depende de factores genéticos, medio ambiente y métodos de cultivo (Brisibe et al., 2009).

De igual manera, existen diferencias al utilizar diversos protocolos para determinar digestibilidades, ya que estos requieren información como estimativas de consumo voluntario, digestibilidad y valor nutricional de las fuentes forrajeras (Ceballos et al., 2008) y esto depende de las características de los animales utilizados (edad, peso, sexo, raza y especie, entre otras).

\section{Conclusiones}

- A pesar del valor nutricional que presenta la Moringa oleifera y el buen comportamiento en la alimentación de rumiantes, los estudios de carácter científico debidamente realizados son muy pocos. Se requiere que los investigadores compartan la información generada y que los artículos contengan toda la información requerida para poder replicar los experimentos.

- La variabilidad en los valores encontrados en los diferentes estudios con relación a la calidad bromatológica de Moringa oleifera se pueden deber a condiciones agroclimáticas diversas o a características fenológicas de la planta, y estado de madurez.

- Las condiciones en que se desarrollan los estudios con diversidad en el tipo de material usado, las mezclas elaboradas, las metodologías y técnicas utilizadas, constituyen factores de variación en los resultados encontrados.

\section{Agradecimientos}

Los autores agradecen a la Universidad del Tolima por la comisión de estudios otorgada y a la Universidad de Caldas por la asesoría recibida de sus investigadores.

\section{Literatura Citada}

1. Adegun MK, Aye PA, Dairo FAS. 2011. Evaluation of Moringa oleifera, Gliricidia sepium and Leucaena leucocephala-based multinutrient blocks as feed supplements for sheep in South Western Nigeria. Agric Biol J N Am 2: 1395-1401. doi:10.5251/ abjna.2011.2.11.1395.1401

2. Akinfemi A, Adesanya AO, Aya VE. 2009. Use of an in vitro gas production technique to evaluate some Nigerian feedstuff. Am Eurasian J Sci Res 4: 240245.

3. Alexander G, Singh B, Sahoo A, Bhat TK. 2008. In vitro screening of plant extracts to enhance the efficiency of utilization of energy and nitrogen in ruminant diets. Anim Feed Sci Tech 145: 229-244. doi:10.1016/j.anifeedsci. 2007.05.036

4. Almanza AJJ, Espinoza DJR, Rocha $L$, Reyes-Sánchez $N$, MendietaAraica B. 2013. Degradabilidad ruminal 
del follaje de Moringa oleifera a tres diferentes edades de rebrote. Calera 13(21): 76-81.

5. Al-Masri MR. 2003. An in vitro evaluation of some unconventional ruminant feeds in terms of the organic matter digestibility, energy and microbial biomass. Trop Anim Health Prod 35: 155167. doi: 10.1023/A:1022877603010

6. Aregheore EM. 2002. Intake and digestibility of Moringa oleifera-batiki grass mixtures by growing goats. Small Ruminant Res 46: 23-28. doi: 10.1016/ S0921-4488(02)00178-5

7. Astuti DA, Baba AS, Wibawan IWT. 2011. Rumen fermentation, blood metabolites, and performance of sheep fed tropical browse plants. Media Peternakan 34: 201-206. doi: 10.5398/ medpet.2011.34.3.201

8. Ben Salem H, Makkar HPS. 2009. Defatted Moringa oleifera seed meal as a feed additive for sheep. Anim Feed Sci Tech 150: 27-33. doi: 10.1016/ j.anifeedsci.2008.07.007

9. Brisibe EA, Umoren UE, Brisibe F, Magalhaes PM, Ferreira JFS, Luthria D, Wu X, Prior RL. 2009. Nutritional characterization and antioxidant capacity of different tissues of Artemisia annua L. Food Chem 115: 1240-1246. doi: 10.1016/j.foodchem. 2009.01 .033

10. Carballo N. 2011. Moringa oleifera Lam. Árbol de la vida. La Habana: CENPALAB. 12 p.

11. Ceballos A, Noguera RR, Bolívar DM, Posada SL. 2008. Comparación de las técnicas in situ de los sacos de nylon e in vitro (Daisy $\left.{ }^{\mathrm{II}}\right)$ para estimar la cinética de degradación de alimentos para rumiantes. Livest Res Rural Dev 20(7). [Internet]. Disponible en: http:// www.lrrd.org/lrrd20/7/ceba20108.htm

12. Cone JW, Van Gelder AH. 1999: Influence of protein fermentation on gas production profiles. Anim Feed Sci Technol 76: 251-264. doi: 10.1016/S03778401(98)00222-3
13. Cornelissen JHC, Lavorel, S, Garnier E, Diaz S, Buchmann N, Gurvich DE, Reich PB, et al. 2003. A handbook of protocols for standardised and easy measurement of plant functional traits worldwide. Aust J Bot 51: 335-380. doi:10.1071/BT02124

14. Falasca S. 2008. Las especies del género Jatropha para producir biodiesel. Redesma 19 p. [Internet]. Disponible en: http://uniciencia.ambientalex.info/ infoCT/Espgenjatprobioar.pdf

15. Falasca S, Bernabé MA. 2008. Potenciales usos y delimitación del área de cultivo de Moringa oleifera en Argentina. Redesma. [Internet]. Disponible en: http://api.ning.com/files/ zlToq5jvxOn7za5bxe0jeeDaRlh0Qk15gz 8YZv0M14Y26PAYaa9v2U yA9wryK zDMyfI* GioYzU p8n4wbrjA* 6kkIdY Meaxm2/Moringa investigacin Argentina. pdf

16. Ferreira F, Urrutia G, Alonso-Coello P. 2011. Revisiones sistémicas y metaanálisis: bases conceptuales e interpretación. Rev Esp Cardiol 64: 688-696. doi: 10.1016/j.recesp.2011.03.029

17. Foidl N, Mayorga L, Vásquez W. 2011. Utilización del marango (Moringa oleifera) como forraje fresco para ganado. En: Conferencia electrónica de la FAO sobre «Agroforestería para la producción animal en Latinoamérica». [Internet]. Disponible en: http:// www.fao.org/livestock/agap/frg/ agrofor $1 /$ foidl16.htm

18. Folkard G, Sutherland J. 1996. Moringa oleifera un árbol con enormes potencialidades. Agroforestería en las Américas. [Internet]. Disponible en: ftp:/ /ftp.fao.org/docrep/nonfao/LEAD/ X6324S/X6324S00.pdf

19. Frutos P, Hervas G, Ramos G, Giraldez FJ, Montecon AR. 2002. Condensed tannin content of several shrub species from a mountain area in northern Spain, and its relationship to various indicators of nutritive value. Anim Feed Sci Tech 95: 215-226. doi: 10.1016/ S0377-8401(01)00323-6 
20. Getachew G, Makkar HPS, Becker K. 2000. Effect of polyethylene glycol on in vitro degradability and microbial protein synthesis from tannin-rich browse and herbaceous legumes. $\mathrm{Br} \mathrm{J}$ Nutrit 84: 73-83.

21. Getachew G, Robinson PH, DePeters EJ, Taylor SJ. 2004. Relationships between chemical composition, dry matter degradation and in vitro gas production of several ruminant feeds. Anim Feed Sci Technol 111: 57-71. doi: 10.1016/S0377-8401(03)00217-7

22. Gómez Ortega OR, Amaya Rey MC. 2013. ICrESAI-IMeCI: instrumentos para elegir y evaluar artículos científicos para la investigación y la práctica basada en evidencia. Aquichan 13: 407420.

23. Gutiérrez PM. 2012. Determinación de la tasa de degradación ruminal del follaje de Marango (Moringa oleifera) usando la técnica in sacco en vacas Reyna. Finca Santa Rosa, Managua, Nicaragua. Tesis de grado. Managua, Nicaragua: Universidad Nacional Agraria. $35 \mathrm{p}$.

24. Higgins JPT, Green S. 2011. Manual Cochrane de revisiones sistemáticas de intervenciones. v. 5.1.0 [Internet]. Disponible en: https://es.cochrane.org/sites/ es.cochrane.org/files/uploads/ Manual_Cochrane_510_reduit.pdf

25. Hoffmann EM, Muetzel S, Becker K. 2003. Effects of Moringa oleifera seed extract on rumen fermentation in vitro. Arch Tierernahr 57: 65-81. doi: 10.1080/0003942031000086617

26. Jelali R, Ben Salem H. 2014. Daily and alternate day supplementation of Moringa oleifera leaf meal or soyabean meal to lambs receiving oat hay. Livest Sci 168: 84-88. doi: 10.1016/j.livsci. 2014.07.005

27. Lam Kim Yen, Luи Huи Manh, Bach Tuan Kiet, Nguyen Nhut Xuan Dung, Tran Phung Ngoi. 2007. Effect of Moringa oleifera on performance and nitrogen utilization of growing goats. En: MEKARN Regional Conference 2007.
[Internet]. Disponible en: http:// www.mekarn.org/prohan/manh.htm

28. Luи Нuи Manh, Nguyen Nhut Xuan Dung, Tran Phung Ngoi. 2005. Introduction and evaluation of Moringa oleifera for biomass production and as feed for goats in the Mekong Delta. Livest Res Rural Dev 17(9). [Internet]. Disponible en: http://www.lrrd.org/ lrrd17/9/manh17104.htm

29. Makkar HPS, Becker K. 1996. Nutritional value and antinutritional components of whole and etanol extracted Moringa oleifera leaves. Anim Feed Sci Tech 63: 211-228. doi: 10.1016/S0377-8401(96)01023-1

30. Martín C, Martín G, García A, Fernández T, Hernández E, Puls J. 2013. Potenciales aplicaciones de Moringa oleifera. Una revisión crítica. Pastos y Forrajes 36: 137-149.

31. Melesse A, Bulang M, Kluth H. 2009. Evaluating the nutritive values and in vitro degradability characteristics of leaves, seeds and seedpods from $M$. stenopetala. J Sci Food Agric 89: 281287. doi: $10.1002 /$ jsfa. 3439

32. Melesse A, Steingass H, Boguhn J, Rodehutscord M. 2013. In vitro fermentation characteristics and effective utilisable crude protein in leaves and green pods of Moringa stenopetala and Moringa oleifera cultivated at low and mid-altitudes. J Anim Physiol Anim Nutr (Berl) 97: 537-546. doi: 10.1111/ j.1439-0396.2012.01294.x

33. Mendieta-Araica B, Spörndly R, Reyes-Sánchez N, Spörndly E. 2011. Moringa (Moringa oleifera) leaf meal as a source of protein in locally produced concentrates for dairy cows fed low protein diets in tropical areas. Livest Sci 137: 10-17. doi: 10.1016/j.livsci. 2010.09 .021

34. Montejo IL, López O, Sánchez T, Muetzel S, Becker K, Lamela L. Efecto del nivel de inclusión de soya en la digestibilidad in vitro de la harina de piscidium de Moringa oleifera. Pastos y Forrajes 35: 197-204. 
35. Moyo B, Masika PJ, Muchenje V. 2014. Effect of feeding moringa (Moringa oleifera) leaf meal on the physico-chemical characteristics and sensory properties of goat meat. S Afr J Anim Sci 44: 64-70.

36. Murro JK, Muhikambele VRM, Sarwatt SV. 2003. Moringa oleifera leaf meal can replace cottonseed cake in the concentrate mix fed with Rhodes grass (Chloris gayana) hay for growing sheep. Livest Res Rural Dev 15(11). [Internet]. Disponible en: http://www.1rrd.org/1rrd15/11/ murr1511.htm

37. Nouala FS, Akinbamijo OO, Adewumi A, Hoffman E, Muetzel S, Becker K. 2006. The influence of Moringa oleifera leaves as substitute to conventional concentrate on the in vitro gas production and digestibility of groundnut hay. Livest Res Rural Dev 18: 121. [Internet]. Disponible en: http:// www.lrrd.org/lrrd18/9/noua18121.htm

38. Pacheco RM. 2006. Análisis del intercambio de plantas entre México y Asia de los siglos XVI al XIX. Tesis de Maestría. México DF: Univ Nacional Autónoma de México. $254 \mathrm{p}$.

39. Patra AK. 2010. Aspects of nitrogen metabolism in sheep-fed mixed diets containing tree and shrub foliages. $\mathrm{Br} \mathrm{J}$ Nutrit 103: 1319-1330. doi: 10.1017/ S0007114509993254

40. Pedraza R, Pérez S, González M, González E, León M, Espinosa E. 2013. Indicadores in vitro del valor nutritivo de Moringa oleifera en época de seca para rumiantes. Rev Prod Anim 25 (Especial).

41. Pérez A, Sánchez N, Amerangal N, Reyes F. 2010. Características y potencialidades de Moringa oleifera, Lamark. Una alternativa para la alimentación animal. Pastos y Forrajes 33: 1-16.

42. Pérez AN, Ibrahim M, Villanueva C, Skarpe C, Cuerin H. 2013. Diversidad forrajera tropical 2. Rasgos funcionales que determinan la calidad nutricional y preferencia de leñosas forrajeras para su inclusión en sistemas de alimentación ganadera en zonas secas. Agroforestería en las Américas 50: 44-52.

43. Ramachandran C, Peter KV, Gopalak-rishnan PK. 1980. Drumstick (Moringa oleifera): a multipurpose Indian vegetable. Econ Bot 34: 276-283.

44. Reich PB, Wright IJ, Cavender-Bares J, Craine JM, Oleksyn J, Westoby KM, Walters MB. 2003. The evolution of plant functional variation: traits, spectra, and strategies. Int. J Plant Sci 164 (Suppl): S143-S164.

45. Reyes SN. 2006. Moringa oleifera and Cratylia argentea: potential fodder species for ruminants in Nicaragua. $\mathrm{PhD}$ Thesis. Uppsala, Sweden: Swedish University of Agricultural Sciences. $51 \mathrm{p}$.

46. Rodríguez R. 2011. Alimentación de vacas lecheras con Moringa oleifera, fresco o ensilado y su efecto sobre la producción, composición y calidad de leche. Tesis de Maestría. Managua, Nicaragua: Univ Nacional Agraria. 35 p.

47. Rodríguez R, González N, Alonso J, Domínguez M, Sarduy L. 2014. Valor nutritivo de harinas de follaje de cuatro especies arbóreas tropicales para rumiantes. Rev Cub Cienc Agríc 48: 371-378.

48. Sánchez NR, Spörndly E, Ledin I. 2006. Effect of feeding different levels of foliage of Moringa oleifera to creole dairy cows on intake, digestibility, milk production and composition. Livest Sci 101: 24-31. doi: 10.1016/j.livprodsci. 2005.09.010

49. Sarwatt SV, Kapange SS, Kakengi $A M V .2002$. Substituting sunflower seedcake with Moringa oleifera leaves as a supplemental goat feed in Tanzania. Agroforest Syst 56: 241-247. doi: 10.1023/A:1021396629613

50. Sarwatt SV, Milang'ha MS, Lekule FP, Madalla N. 2004. Moringa oleifera and cottonseed cake as supplements for smallholder dairy cows fed Napier grass. Livest Res Rural Dev 16(6). [Internet]. Disponible en: http:// www.lrrd.org/lrrd16/6/sarw16038.htm 
51. Soliva CR, Kreuzer M, Foidl N, Foidl G, Machmüller A, Hess HD. 2005. Feeding value of whole and extracted Moringa oleifera leaves for ruminants and their effects on ruminal fermentation in vitro. Anim Feed Sci Tech 118: $47-$ 62. doi: 10.1016/j.anifeedsci.2004.10.005 